\title{
Producción de cuatro variedades de aguacate (Persea americana Mill.) en el noroeste de Puerto Rico
}

\section{Karla Verónica Jaramillo Jiménez}

\author{
Máster en Ciencias en Horticultura \\ IKIAM EP, Técnico de proyectos, Ecuador \\ Email: karlajaramillo88@gmail.com \\ ORCID: https://orcid.org/0000-0002-0300-1821
}

DOI:

Recepción: 30/07/2021 Aceptación: 31/08/2021

\section{Resumen}

El aguacate es un cultivo de importancia económica en Puerto Rico. Sin embargo, la información documentada es limitada en variedades como: 'Ávila', 'Candelaria,' 'Domínguez' y 'Goya', establecidas en la Estación Experimental Agrícola del municipio de Isabela al noroeste de ese país (coordenadas 18³0’00” latitud norte, $67^{\circ} 00^{\prime} 00^{\prime \prime}$ longitud oeste a una altitud de $128 \mathrm{msnm}$, con promedios anuales de temperatura de $25^{\circ} \mathrm{C}$, precipitación de $1592 \mathrm{~mm}$ y una humedad relativa de $70 \%$ ).

En consecuencia, se determinaron fechas de corte, número y peso de frutos totales y comerciales; distribución de frutos por calibre y producción total durante dos ciclos de producción. En el año 2016, el corte no obtuvo diferencias significativas: inició en septiembre y las variedades con mayor producción fueron 'Candelaria,' 'Domínguez' y 'Ávila'; mientras que 'Goya' presentó la menor producción. En el año 2017, el corte obtuvo diferencias significativas: inició en julio y terminó en agosto por efecto de los huracanes Irma y María.

La variedad más tardía fue 'Goya', que perdió $25 \%$ de frutos por los huracanes; en el caso de 'Candelaria' hubo un corte y perdió un $67 \%$ de frutos potencialmente comerciales; mientras que, la variedad 'Ávila' tuvo una pérdida de $18 \%$ y 'Domínguez' 13\%. Los calibres de fruto comercial tres (500 g a 699 g) y cuatro (300 g a $499 \mathrm{~g}$ ) predominaron. Las variedades que alcanzaron el mayor calibre fueron 'Candelaria' con $1070 \mathrm{~g}$ y 'Ávila’ con 990 g; los calibres medianos fueron para 'Domínguez’ con 760 g y ‘Goya’ con 565 g.

Palabras clave: Variedades, producción, frutos, corte, calibres.

\begin{abstract}
Avocado is an economically important crop in Puerto Rico. However, the documented information is limited in varieties such as: 'Ávila', 'Candelaria', 'Domínguez' and 'Goya', established in the Agricultural Experiment Station of the municipality of Isabela in the northwest of that country (coordinates $18^{\circ} 30^{\prime} 00^{\prime}$ north latitude, $67^{\circ} 00^{\prime} 00^{\prime}$ west longitude at an altitude of 128 meters above sea level, with annual averages of temperature of $25^{\circ} \mathrm{C}$, precipitation of $1592 \mathrm{~mm}$ and a relative humidity of $70 \%$ ).

Consequently, cutting dates, number and weight of total and commercial fruits were determined; distribution of fruits by size and total production during two production cycles. In 2016, the cut did not obtain significant differences: it started in September and the varieties with the highest production were 'Candelaria', 'Domínguez' and 'Ávila'; while 'Goya' presented the lowest production. In 2017, the cut obtained significant differences: it began in July and ended in August due to the effect of hurricanes Irma and María.

The latest variety was 'Goya', which lost $25 \%$ of its fruit due to the hurricanes; in the case of 'Candelaria' there was a cut and it lost $67 \%$ of potentially commercial fruits; while, the variety 'Ávila' had a loss of $18 \%$ and 'Domínguez ' $13 \%$. The commercial fruit sizes three (500 g to $699 \mathrm{~g}$ ) and four ( $300 \mathrm{~g}$ to $499 \mathrm{~g}$ ) predominated. The varieties that reached the highest caliber were 'Candelaria' with $1070 \mathrm{~g}$ and 'Ávila' with $990 \mathrm{~g}$; the medium sizes were for 'Domínguez' with $760 \mathrm{~g}$ and 'Goya' with $565 \mathrm{~g}$.
\end{abstract}

Key words: Varieties, production, fruits, cut, sizes. 


\section{Introducción}

El aguacate es un cultivo tradicional en Puerto Rico, con un consumo de 75 mil quintales de aguacate anualmente; pero se producen mucho menos de 9 mil quintales en la actualidad (Departamento de Agricultura de Puerto Rico, 2019). En la isla se cultivan diferentes variedades de aguacate, sobre todo de raza o tipo Antillano y Antillano x guatemalteco.

Muchas de estas variedades han sido descritas someramente, y rara vez se ha comparado con rigor estadístico la productividad de variedades. Entre las variedades cultivadas en Puerto Rico están 'Ávila’ y 'Candelaria', que fueron seleccionadas en la isla (EEA, 1998). La información documentada sobre ambas es escasa y mayormente anecdótica, con poca información basada en experimentos o en descriptores morfológicos usados internacionalmente. Además, existen otras variedades prometedoras (aún no usadas comercialmente) como 'Domínguez' y 'Goya' que no tienen producción documentada, pero se sabe que fueron seleccionadas de árboles puertorriqueños; y que, en los primeros años del siglo XXI, eran parte de una colección de aguacates - ya inexistente - en la finca de Juana Díaz de la Estación Experimental Agrícola del Recinto Universitario de Mayagüez de la Universidad de Puerto Rico.

Observaciones realizadas en el municipio de Isabela, Puerto Rico, durante las temporadas de corte de los años 2013, 2014, 2015 y 2016 muestran que tanto 'Domínguez' como 'Goya' tienen época de corte similar a 'Ávila' y 'Candelaria' (generalmente de agosto a octubre); y por tanto, en esos meses las cuatro variedades competirían por el mercado. El aguacate es un cultivo con potencial de crecimiento para la expansión de áreas de siembra, por lo que es necesario proveer a los agricultores de información comparativa-objetiva sobre la producción de variedades de aguacate en la isla, en lugar de depender de información anecdótica. Con esa información, los agricultores presentes y potenciales podrán tomar decisiones bien informadas sobre cuales variedades plantar, según los propósitos que tengan.

\section{Materiales y Métodos}

Se analizaron árboles de aguacate Persea americana de raza Antillana de las variedades 'Ávila', 'Candelaria,' 'Domínguez' y 'Goya' injertadas en el patrón 'Gripiña', que fueron establecidas en el año 2009 junto con otras once variedades, bajo un sistema de plantación de marco real con una superficie de $7.5 \mathrm{~m} 2$, con un área total de $1,372.5 \mathrm{~m} 2$. El huerto fue dispuesto en un diseño de bloques completos al azar con cuatro repeticiones, teniendo ocho árboles por variedad y un total de 120 ejemplares; para los análisis estadísticos se utilizó un diseño completamente al azar en donde se evaluaron ocho árboles por variedad.

Para determinar el porcentaje de frutos retenidos, cada semana hasta culminar el corte, se contabilizó el número de frutos encontra- 
dos en el suelo cerca del árbol. Esta acción se realizó con todos los árboles de cada una de las cuatro variedades. Posteriormente, por diferencia se obtuvo el número de frutos retenidos y perdidos por árbol con la siguiente ecuación: Porcentaje de frutos retenidos $=\frac{\text { Frutos retenidos }}{\text { Total de frutos }(\text { caídos }+ \text { retenidos })} \times 100$

La distribución temporal de cortes se determinó registrando desde el inicio del primer corte hasta el corte de todos los frutos comerciales y no comerciales de cada variedad, considerando que hubo cortes por tres meses en cada año evaluado. Consecuentemente, se realizó la distribución de corte en el tiempo para establecer las semanas de mayor y menor número de frutos cortados por variedad.

Para determinar el número de frutos cortados por variedad, se registró el número de frutos comerciales y no comerciales cortados a lo largo de todo el período de corte. Para determinar el número y peso de frutos comerciales y no comerciales se pesaron y contaron aquellos frutos que cumplían con las normas establecidas por la Organización de las Naciones Unidas para la Alimentación $(\mathrm{FAO})^{1}$ (2013) para la raza Antillana, en donde incluyen características mínimas de calidad, así como también un peso superior a 170 gramos al momento del corte, usando una balanza digital.

La clasificación por calibres se realizó de acuerdo con el peso en gramos obtenido en la madurez fisiológica. Esto permitió detectar la distribución de los frutos de las cuatro variedades con los siguientes parámetros:

\section{Tabla 1}

Distribución de los frutos de las cuatro variedades por calibre

\begin{tabular}{lc}
\hline Clasificación & Peso en gramos \\
\hline Calibre 1 & $\geq 900 \mathrm{~g}$ 'Comercial' \\
\hline Calibre 2 & $700 \mathrm{~g}-899 \mathrm{~g}$ \\
\hline Calibre 3 & $500 \mathrm{~g}-699 \mathrm{~g}$ \\
\hline Calibre 4 & $300 \mathrm{~g}-499 \mathrm{~g}$ \\
\hline Calibre 5 & $171 \mathrm{~g}-299 \mathrm{~g}$ \\
\hline Calibre 6 & $\leq 170 \mathrm{~g}$ 'No Comercial' \\
\hline
\end{tabular}

La producción de frutos por árbol se determinó por conteo, y se registró el número total de frutos comerciales y no comerciales producidos por variedad, durante todo el ciclo de corte en los años 2016 y 2017.

\section{Resultados y Discusión}

Una vez iniciada la fructificación en los árboles de aguacate, la caída de los frutos fue notable en las cuatro variedades; determinada semanalmente por conteo de frutos recolectados del suelo, que se encontraban bajo la copa de cada árbol. Del total de frutos cua-

1. Dentro del documento, la autora hará referencia a este término mediante su acrónimo. 
jados o formados, la variedad 'Ávila' retuvo el porcentaje más alto con $15.05 \%$; 'Goya' y 'Domínguez' permanecieron con un porcentaje similar de $5.07 \%$ y $4.28 \%$ respectivamente; mientras que la variedad 'Candelaria' presentó la menor cantidad de frutos retenidos con $1.72 \%$.

Durante el periodo de evaluación, el número de frutos caídos fue mayor en las primeras semanas, cuando los frutos tenían tamaños pequeños y eran más sensibles a factores ambientales como el viento y las precipitaciones. Sin embargo, la variedad 'Candelaria' tuvo en la antepenúltima y última fecha valores altos de caída de fruta, debido al paso de los huracanes Irma y María; algunos de los frutos para esas fechas aún no se encontraban en punto de madurez fisiológica.

Independientemente de este factor, las cuatro variedades a partir de la doceava fecha de toma de datos de caída de frutos redujeron considerablemente el número de frutos caídos, permaneciendo en el árbol mayor número de frutos para completar la madurez fisiológica ( ver figura 1).

Las fechas de corte en los años 2016 y 2017 difirieron en inicio, duración y culminación, siendo para el primer año un corte más tardío y para el segundo año el corte más precoz. Para el año 2016, en las variedades 'Ávila' y 'Domínguez', la producción de frutos fue

\section{Figura 1}

Promedio semanal de frutos caídos por árbol durante el periodo de fructificación en cuatro variedades de aguacate.

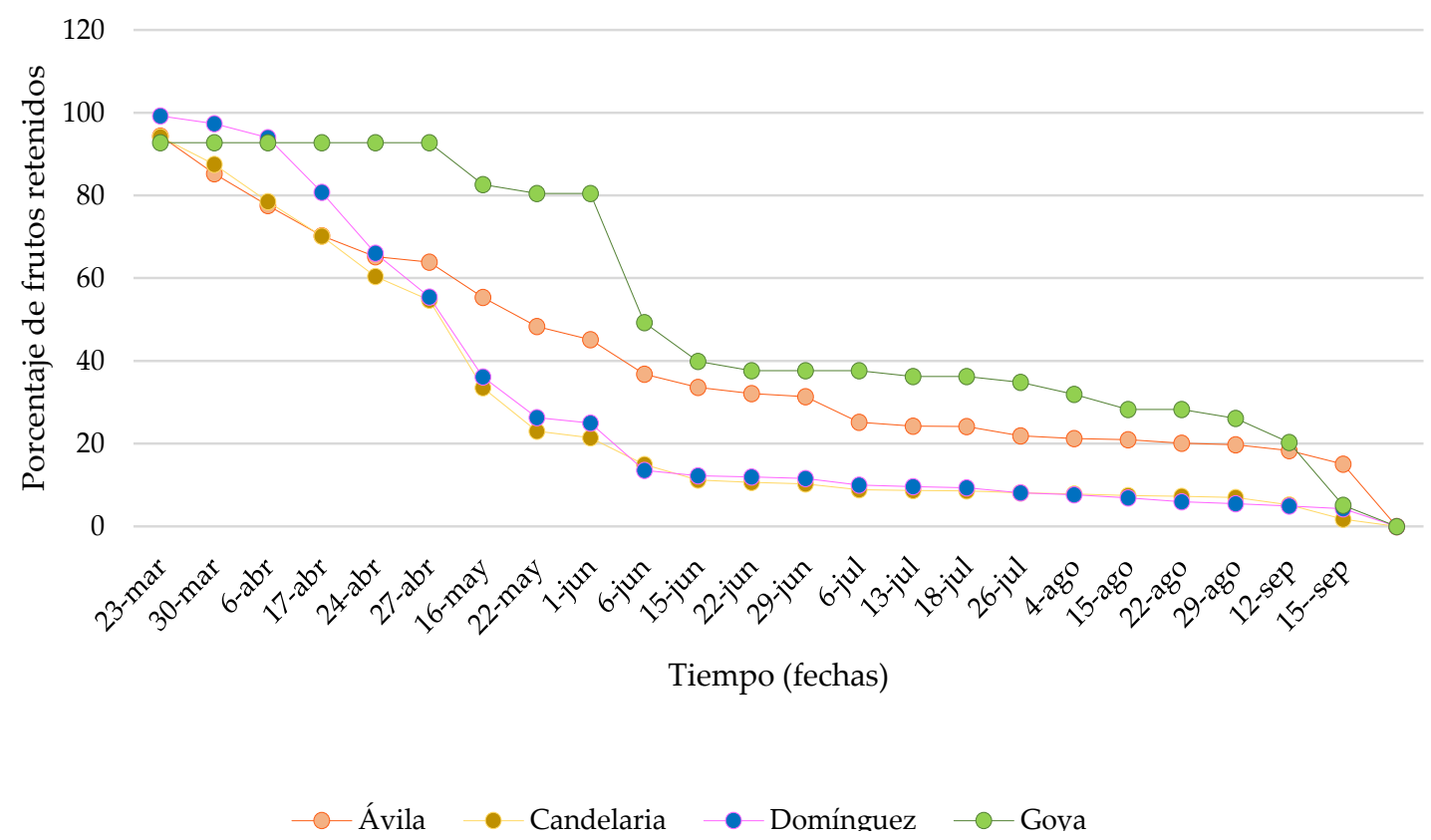


baja por lo que se realizaron dos cortes; pero solo el primero, realizado el 13 de septiembre, concentró toda la producción. En cambio, en la variedad 'Candelaria' se hicieron cuatro cortes, iniciando el 4 de octubre, en donde el número de frutos aumentó considerablemente hasta el último corte.

Para el año 2017, los frutos de las cuatro variedades completaron su madurez fisiológica el 7 de julio y se realizó nueve cortes hasta el 12 de septiembre, fecha previa a la llegada del huracán María. En las fechas analizadas se puede distinguir que la variedad 'Ávila' tuvo un número similar de frutos en cada corte; excepto con un mayor número en la primera. 'Domínguez' tuvo dos fechas de mayor número de frutos cortados, correspondientes a la primera y tercera fecha; para las fechas res- tantes, el número de frutos cortados en 'Domínguez’ fue variable.

Para la variedad 'Candelaria', la mayor cantidad de frutos fue en el primer corte; pero durante las fechas restantes disminuyó con un ligero incremento en la última recolección. Para la variedad 'Goya', en ambos años, solo se realizaron dos cortes en los años 2016 y 2017 con un número de frutos bajo (ver figura 2).

De los cortes, los frutos considerados no comerciales fueron aquellos que presentaron daños mecánicos o lesiones por plagas y enfermedades con más del 25\% del área total de la cáscara del fruto; así como los frutos con pesos inferiores a $170 \mathrm{~g}$ como indica la FAO. Los frutos que no presentaron imperfecciones fueron considerados comerciales.

\section{Figura 2}

Fechas de corte en cuatro variedades de aguacate

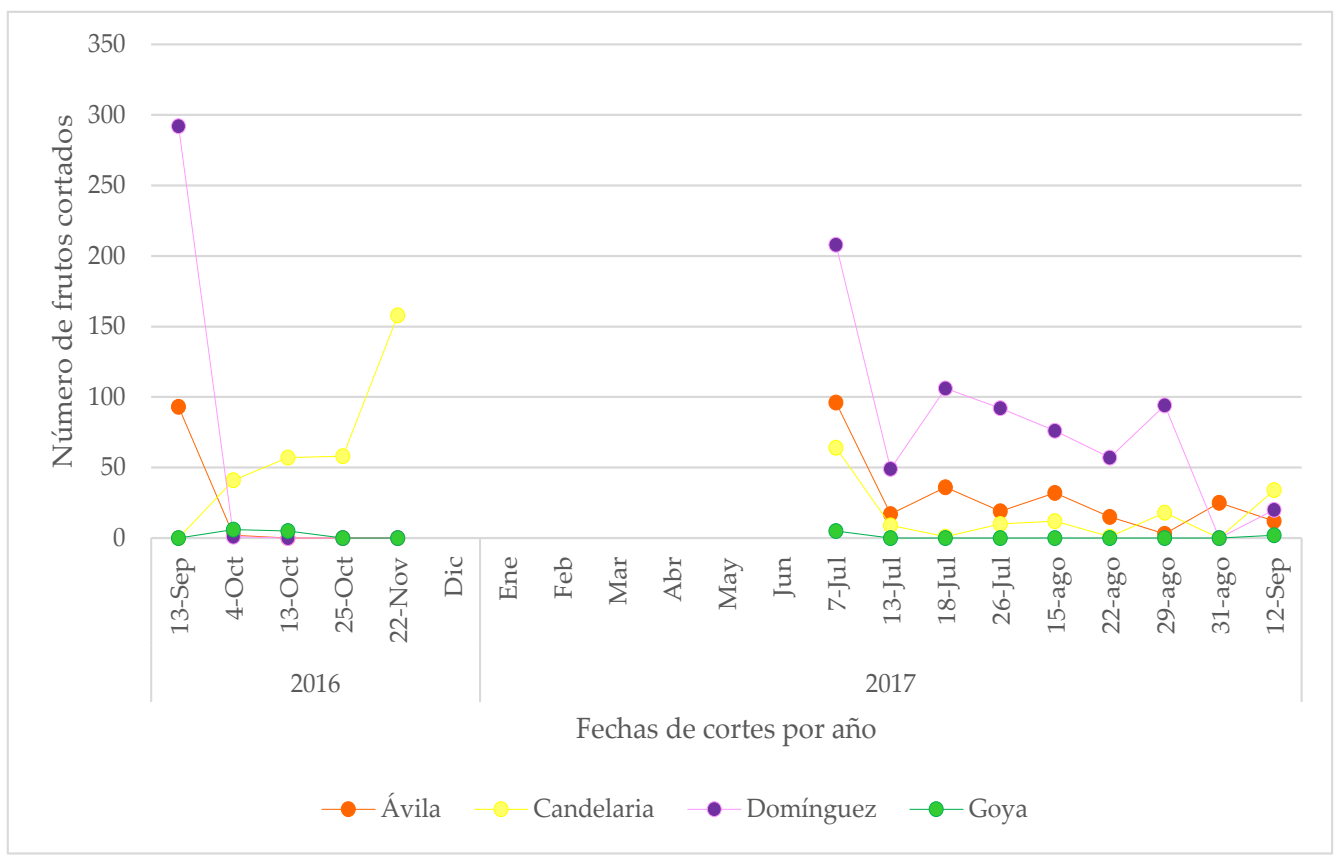


En todas las variedades, el número de frutos comerciales fue mayor al de no comerciales. La variedad 'Candelaria' tuvo el mayor porcentaje de frutos no comerciales con un $23 \%$, seguida de 'Domínguez' con un 16\% (ver tabla 2).

En la variable número de frutos cortados por variedad, no se detectaron diferencias significativas en el corte del año 2016. En cambio, en el año 2017 (ver tabla 3) se detectaron diferencias significativas, donde la variedad 'Domínguez' tuvo el promedio más alto de $87.75 \pm 15.30$ de frutos cortados, mucho más que 'Ávila' con $36.43 \pm 9.62$; o 'Candelaria' con $24.83 \pm 10.20$.

Estos resultados responden a la característica de bienalidad o alternancia productiva de frutales, como el caso de ciertas variedades de aguacate en donde hay años de mayor producción que otros.

Al comparar los años 2016 y 2017 se puede apreciar que, a pesar de los huracanes Irma y

\section{Tabla 2}

Porcentaje, número y peso de frutos comerciales y no comerciales en cuatro variedades de aguacate

\begin{tabular}{|c|c|c|c|c|c|c|c|c|c|c|}
\hline \multicolumn{6}{|c|}{ Frutos comerciales } & \multicolumn{5}{|c|}{ Frutos no comerciales } \\
\hline Variedad & Porcentaje & Número & $\begin{array}{l}\text { Peso } \\
\text { total } \\
(\mathrm{g})\end{array}$ & $\begin{array}{c}\text { Peso } \\
\text { promedio } \\
\text { (g) }\end{array}$ & Calibre & Porcentaje & Número & $\begin{array}{c}\text { Peso } \\
\text { total } \\
(\mathrm{g})\end{array}$ & $\begin{array}{c}\text { Peso } \\
\text { promedio } \\
\text { (g) }\end{array}$ & Calibre \\
\hline 'Ávila' & $91 \%$ & 124 & 81450 & 656.85 & 3 & $9 \%$ & 12 & 6215 & 517.92 & 3 \\
\hline 'Candelaria' & $77 \%$ & 47 & 31005 & 659.68 & 3 & $23 \%$ & 14 & 6945 & 496.07 & 4 \\
\hline ‘Domínguez' & $84 \%$ & 341 & 164141 & 481.35 & 4 & $16 \%$ & 63 & 28384 & 450.54 & 4 \\
\hline 'Goya' & $100 \%$ & 5 & 2565 & 513.00 & 3 & $0 \%$ & 0 & 0 & 0 & - \\
\hline
\end{tabular}

María la producción en el año 2017 fue mayor; aun considerando que parte de la producción de la variedad 'Candelaria' se perdió debido a ellos, y que la variedad 'Goya' prácticamente no tuvo producción en ese año. Otro factor para considerar en esta variable es el número de árboles cortados, ya que en el año 2017 las variedades 'Ávila’ y ‘Candelaria' tuvieron cortes en seis árboles (ver figura 3).

Para la variable clasificación por calibres en la producción total del año 2016, el calibre 3 que considera frutos de 500 a 699 gramos predominó para las variedades 'Ávila' con 48 frutos y 'Domínguez' con 198 frutos. El calibre 4 de 300 a 499 gramos predominó en las variedades 'Candelaria' con 111 frutos y 'Goya' con seis frutos.

Para el año 2017, las variedades 'Ávila’ y ‘Goya’ mantuvieron la predominancia de los calibres con 42 y cinco frutos, respectivamente. La variedad 'Candelaria' cambió de calibre 4 a 3, es 
Tabla 3

Número promedio de frutos y frutos por árbol cortados por año en cuatro variedades de aguacate

\begin{tabular}{lcc|cc}
\hline \multicolumn{1}{c|}{ Año 2016 } & \multicolumn{2}{c}{ Año 2017 } \\
\hline Variedad & Promedio & Árboles cortados & Promedio & Árboles cortados \\
\hline 'Ávila' & $22.67 \pm 10.71 \mathrm{a}$ & 3 & $36.43 \pm 9.62 \mathrm{ab}$ & 6 \\
\hline 'Candelaria' & $39.25 \pm 17.44 \mathrm{a}$ & 8 & $24.83 \pm 10.20 \mathrm{~b}$ & 6 \\
\hline 'Domínguez' & $41.86 \pm 11.89 \mathrm{a}$ & 7 & $87.75 \pm 15.30 \mathrm{a}$ & 8 \\
\hline 'Goya' & $3.67 \pm 0.88 \mathrm{a}$ & 3 & $2.33 \pm 1.33 \mathrm{~b}$ & 3 \\
\hline
\end{tabular}

Nota. Medias con una letra común en la misma columna no son significativamente diferentes ( $\mathrm{p}>0.05$ ), determinado con la prueba de Diferencia Mínima de Tukey.

\section{Figura 3}

Número total de frutos cortados por árbol en cuatro variedades de aguacate

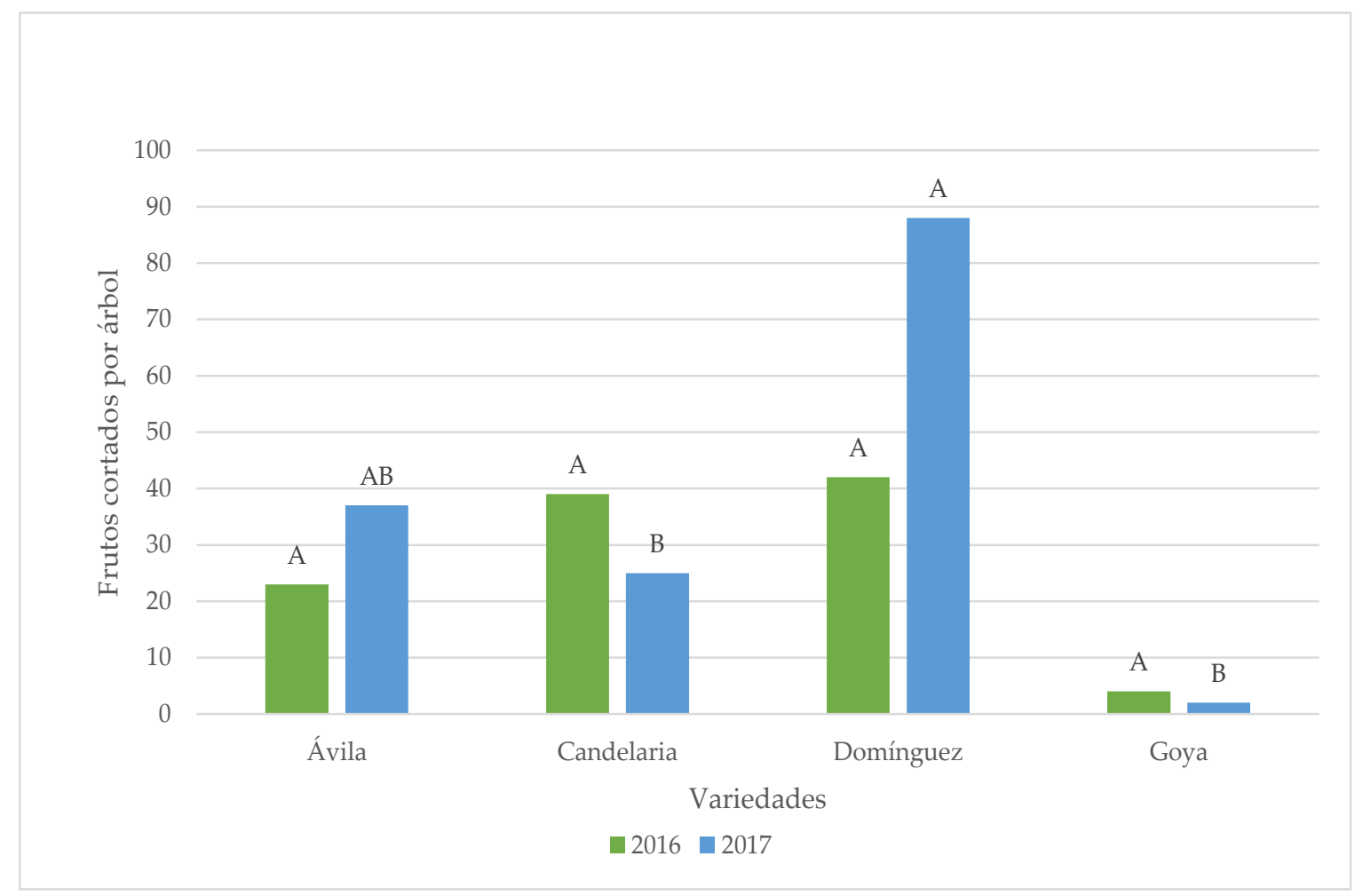

Nota. Medias con una letra común en la misma columna no son significativamente diferentes ( $\mathrm{p}>0.05$ ), determinado con la prueba de Diferencia Mínima de Tukey. 
decir, sus frutos presentaron incremento de tamaño para el calibre predominante con 64 frutos. La variedad 'Domínguez' cambio de calibre 3 a 4; lo que indica que los frutos presentaron reducción de tamaño con una predominancia del calibre 4 de 435 frutos (ver figura 4).

La producción total de los árboles de aguacate fue afectada directamente por los huracanes Irma y María; este último azotó la isla de Puerto Rico el 20 de septiembre de 2017. Según el National Weather Service WFO San Juan (2017), el huracán fue de categoría 4 con vientos sostenidos de $233 \mathrm{~km}$ por hora, entrando por el sureste de la isla y saliendo por la zona noroeste, dejando 1,016 $\mathrm{mm}$ de $1 \mathrm{lu}-$ vias acumuladas.

En la figura 5 se resume para las cuatro variedades el registro de frutos cortados y caídos de forma natural a lo largo de la temporada de crecimiento, desde el inicio de la fructificación (febrero-marzo 2017) hasta la mitad de septiembre 2017; así como también, los frutos caídos durante el paso de los huracanes Irma y María. La información se expresa en porcentaje promedio del número total de frutos contabilizados por árbol a lo largo de la temporada, desde floración hasta el paso de los huracanes.

\section{Figura 4}

Número total de frutos cortados por árbol en cuatro variedades de aguacate

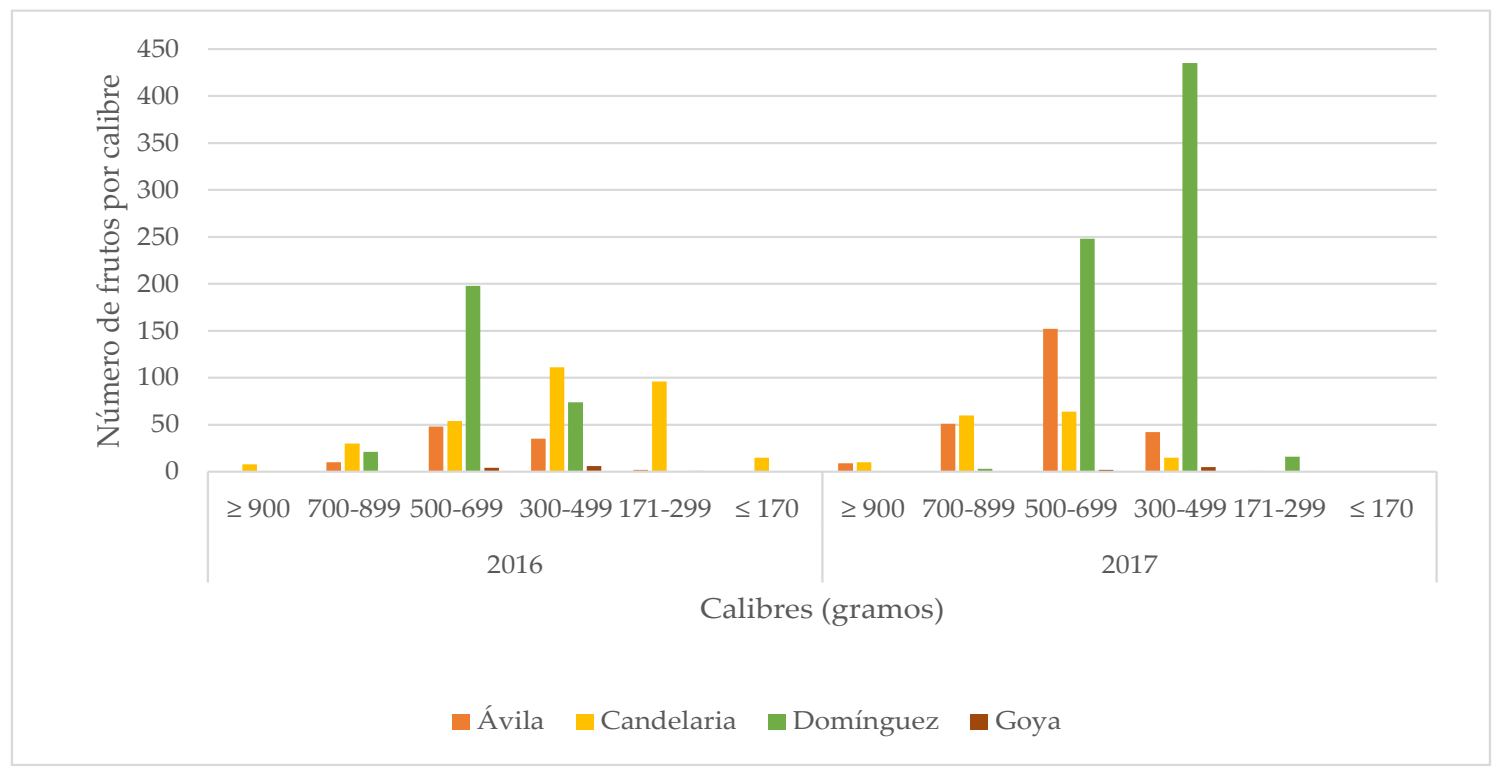




\section{Figura 5}

Porcentaje de frutos perdidos por condiciones atmosféricas, caídas por razones fisiológicas y cortadas en cuatro variedades de aguacate

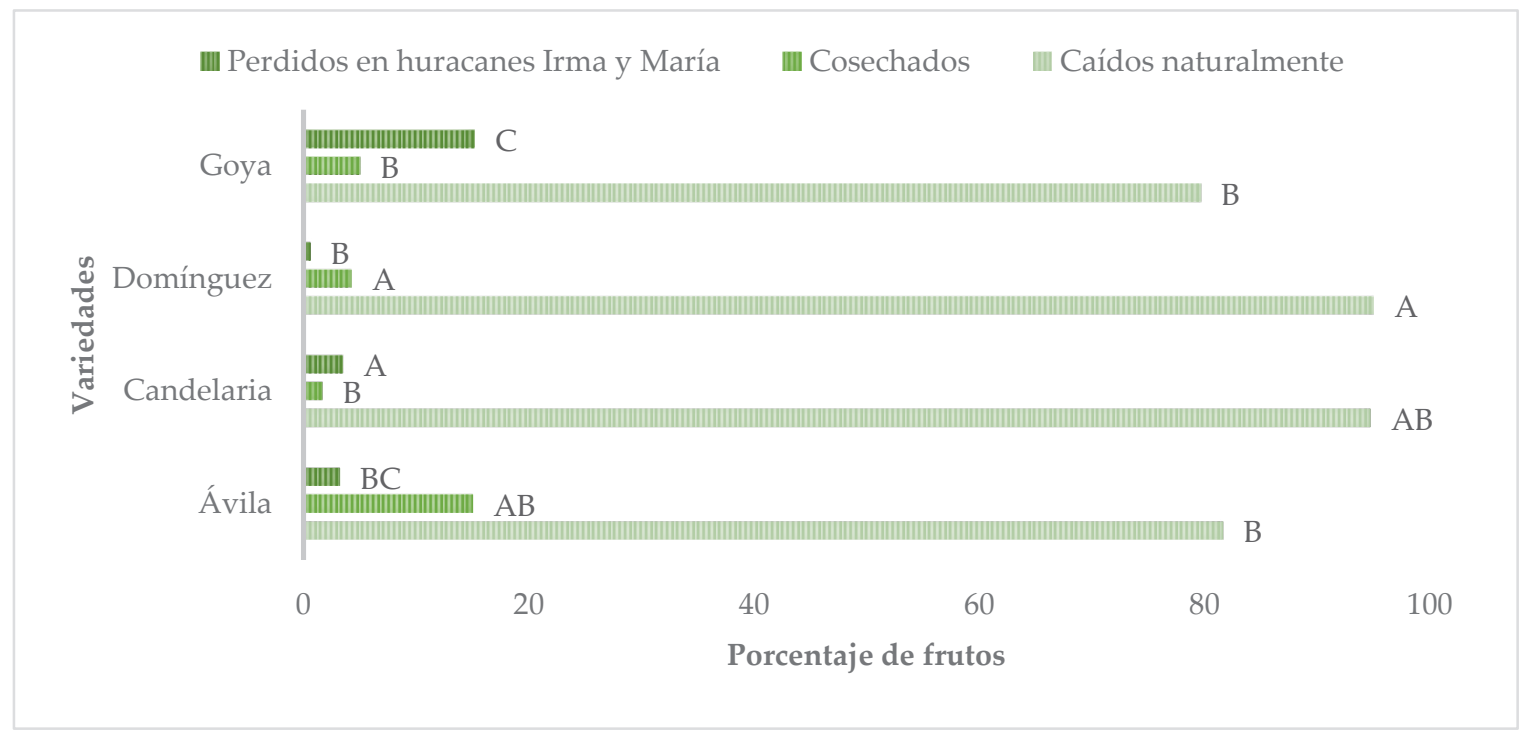

Nota. Medias con una letra común en la misma columna no son significativamente diferentes ( $\mathrm{p}>0.05)$, determinado con la prueba de Diferencia Mínima de Tukey.

En valores absolutos sin efectos de huracanes, considerando los frutos perdidos como comerciales, la producción de las variedades sería: 'Domínguez' 810 frutas, 'Candelaria' 452 frutas, 'Ávila’ 310 frutas y ‘Goya’ 28 frutas. Los valores absolutos con efectos de los huracanes fueron: 'Domínguez' 702 frutos, 'Ávila' 255 frutas, 'Candelaria' 149 frutas y 'Goya' 7 frutas. Estos valores demuestran que la variedad 'Candelaria fue la más afectada porque perdió $67 \%$ de su producción. Las variedades que tuvieron menores pérdidas son 'Ávila' con $18 \%$ y ‘Domínguez' con 13\%.

\section{Conclusiones}

Un árbol de aguacate inicia su etapa productiva en el segundo o tercer año, registrando de dos hasta cuatro floraciones al año, dependiendo de las condiciones climáticas, manejo del huerto y cantidad de fruta presente en el árbol (González et al., 2000). El desarrollo floral del aguacate es iniciado por cambios en las condiciones ambientales y la temperatura es el principal factor involucrado (Álvarez y Salazar 2015). Un árbol de aguacate puede generar de uno a dos millones de flores por 
año. Esto es más de mil veces la cantidad de frutos que puede sustentar; de estas, $0.001 \% \mathrm{y}$ $0.1 \%$ consigue retener el fruto (Cossio-Vargas et al., 2007; Alcaraz et al., 2011).

Una vez los frutos inician el cuajado, la abscisión se puede atribuir a frutos con fallas en el desarrollo de la semilla, aborto del embrión o muerte de la cubierta seminal (Alcaraz et al., 2011). A esto se le suma la preferencia del aguacatero por favorecer al crecimiento vegetativo más que a la producción de frutos (Whiley, 1990); por lo cual solo permanecen en el árbol los frutos más fuertes y vigorosos. Por consiguiente, como mencionan Gazit y Degani (2002), la caída de los frutos aumenta considerablemente por efecto del estrés ambiental, especialmente durante olas de frío o calor. Mientras más joven sea el fruto, mayor es su susceptibilidad a dichos estreses (Romero, 2012).

El diámetro y peso de los frutos es utilizado en Florida, donde cada cultivar debe superar ciertos valores mínimos para ser cortados (Ibar, 1986). Lee y Young (1983), al medir el crecimiento del fruto, determinaron que la porción de descenso en la curva de crecimiento del aguacate determina un punto definitivo, indicando que la madurez fisiológica ha ocurrido, y en muchos casos, esta fecha está relacionada con la fecha de madurez hortícola.

Conocer la predominancia de los calibres en la producción, permite establecer el mercado que se abarcará, ya que como menciona Gardiazabal (2007) el calibre - que está rela- cionado con el peso del fruto - es sumamente importante en la industria. No solo es necesario alcanzar grandes volúmenes de fruta, sino que debe ser de buen tamaño, para que el negocio sea rentable. Pues como asevera Undurraga (2007), el calibre está relacionado con el peso de los frutos y la cantidad de frutos contenidos en un peso de la caja de exportación.

Por esta razón es importante realizar un adecuado manejo del cultivo ya que Cowan (1997) indica que el tamaño final del fruto está limitado por el número de células y no por el tamaño de éstas; por lo que el calibre - al depender del número de células - depende de la división celular, la cual es mayor en la primera etapa del desarrollo del fruto. Una vez cuajado (55 a 60 días es la etapa más sensible en la división celular), cualquier estrés como alta temperatura o radiación puede producir menor calibre.

El tamaño del aguacate se ve afectado fuertemente por la competencia de fotoasimilados y la escasez de carbohidratos afecta al calibre potencial (Wolstenholme y Whiley, 1990). Al reducir la competencia con el crecimiento vegetativo aumenta la localización de materia seca, produciendo mayores rendimientos y un mayor tamaño del fruto. Gardiazabal (2007) señala que el calibre es afectado, principalmente, por la cantidad de corte del árbol. Cerca del 90\% del calibre final de un fruto de aguacate está determinado a las 26 semanas después del cuajado o formación del fruto (Martínez et al., 2003). 


\section{Referencias}

Alcaraz, M.; Rodrigo, J. y Hormaza, J. (2011). Implications of starch content in the flower at anthesis on final fruit set in avocado. En: Memorias VII World Avocado Congress. Cairns, Australia, p. 7.

Álvarez-Bravo, A. y Salazar-García, S. (2015). Validación de un modelo de predicción del desarrollo floral del aguacateく Hass` en Michoacán, México. In VIII Congreso Mundial de la Palta. Lima, Perú, pp. 380-385.

Cossio-Vargas, L. E.; Salazar-García, S.; González-Durán, I. J. L. y Medina-Torres, R. (12-17 noviembre 2007). Modelos de predicción de la determinación irreversible a la floración en los aguacates 'Booth-8' y 'Choquette'. Memorias 3d-127, VI Congreso Mundial de la Palta-Aguacate-Avocado. Viña del mar, Chile. p. 11.

Cowan, A. (1997). Why are small Hass fruit small? South African Avocado Growers' Association Yearbook. 20; 52-54.

Departamento de Agricultura de Puerto Rico (2019). Estadísticas agrícolas de 2018-2019.

EEA (Estación Experimental Agrícola) (1998). Conjunto tecnológico para la producción de aguacates. Recinto Universitario de Mayagüez, Colegio de Ciencias Agrícolas. Estación Experimental Agrícola, Boletín 112. Rio Piedras, Puerto Rico. 23 páginas.

FAO (Food and Agriculture Organization of the United Nations) (2013). World Banana Forum. http://www.fao.org/wbf/en/

Gardiazábal, F.; Mena, F y Magdahl, C. (2007). Efecto de la fertilización en base a N-P-K-CaMg-B-Zn en palto (Persea americana Mill.) cv. Hass sobre su desarrollo, productividad y postcorte de la fruta. P. 12. En: Proceedings VI World Avocado Congress. Viña Del Mar, Chile.

Gazit, S. y Degani, C. (2002). Capítulo 5: Reproductive Biology. pp. 103-131. En: Whiley, A., Schaffer, B.; Wolstenholme, B. (ed.). The avocado, botany, production and uses. Primera edición. CABI Publishing, Londres.

González, G. L. G.; Sánchez, C. I. F.; Ortiz, M. F. P. y Rojas, X. A. A. (2018). producción de aguacate hass una alternativa para el departamento del huila. Crecer Empresarial: Journal of Management and Development, (02), 7-15. 
Ibar, L. (1986). Cultivo del aguacate, chirimoyo, mango, papayo. 3a Edition. Barcelona. Edit. Aedos. España, p. 175

Lee, S. y Young, R. (1983). Growth measurement as an indication of avocado maturity. J. Amer. Soc. Hort. Sci. 108(3); 395-397.

Martínez, R.; Martínez, J.; Martínez-Valero, R. y Martínez, J. (2003). Contribución al estudio de la evolución del crecimiento del fruto del cvr Hass (Persea americana Mill.) con respecto al tiempo en las condiciones ecológicas del área de Motril (Granada, España). Proceding $V$ World Avocado Congress, 257-261.

Romero, M.; Rebolledo, A. y Cayón, D. (2012). Comportamiento fisiológico del aguacate (Persea americana Mill.) Variedad Lorena en la zona de Mariquita, Tolima. [Tesis para optar a Master en Ciencias en Fisiología de cultivos, Universidad Nacional de Colombia]

Undurraga, P., Olaeta, J. A. y Bontá, A. (2007). Evolución de la madurez de frutos del cultivar Isabel (Persea americana Mill.), injertados sobre patrón Mexícola. In Proceedings VI World Avocado Congress. Viña Del Mar, Chile, pp. 45-58.

Whiley, A. (1990). Interpretación de la fenología y fisiología del palto para obtener mayores producciones. Australia.

Wolstenholme, A. y Whiley, A. (1990). Prospects for vegetative reproductive growth manipulation in avocado trees. South African Avocado Growers' Association Yearbook 16; 21-24. 